\section{Debut de linfoma Hodgkin con clínica de compresión medular}

\section{Sr. Director:}

Las complicaciones neurológicas que pueden acompañar a la enfermedad de Hodgkin son poco frecuentes. De entre ellas la compresión medular, por invasión del espacio epidural a partir de adenopatías de la región lumbar, es una situación poco común, algo más frecuente en linfomas no Hodgkin sobre todo de alto grado, y como presentación inicial es rara (1-4). Los linfomas se presentan clínicamente como tumores extramedulares, principalmente de situación extradural, provocando un síndrome compresivo que afecta primero a las raíces y luego a la médula. La afectación radicular provoca intenso dolor que empeora con maniobras de Valsalva, pueden existir parestesias y disminución de la sensibilidad. Suelen afectarse niveles medulares torácicos3. La compresión medular se caracteriza por la clínica motora con espasticidad y exaltación de los reflejos por debajo de la lesión. Presentamos un caso de linfoma de Hodgkin con debut clínico de compresión medular:

Paciente de 45 años, mujer, natural de Guinea Ecuatorial, con antecedentes patológicos de paludismo, último episodio hace un año tratado con quinina; fiebre tifoidea e intervenida de hernia inguinal bilateral hace 5 años; menopaúsica; alérgica a cloroquina. No consumidora de tóxicos ni ha tenido relaciones sexuales de riesgo.

La paciente fue intervenida de una adenopatía axilar izquierda, en su país un año antes de iniciarse la clínica, que fue informada anatomopatológicamente como inespecífica. Viaja a España al presentar clínica subsiguiente para estudio y tratamiento al vivir una hermana en nuestra ciudad.

Una semana antes del ingreso comenzó con dolor en región dorsolumbar con irradiación por ambas extremidades inferiores, con impotencia funcional y disestesias, no controlado con AINEs, de forma concomitante presentó episodios intermitentes de fiebre de hasta $39^{\circ} \mathrm{C}$, pérdida de peso no ponderada e hiporexia.

En la exploración destacaban adenopatías laterocervicales y axilares izdas, duras, no dolorosas, desplazables. Hepatomegalia importante dolorosa y esplenomegalia. Dolor a la palpación de las apófisis espinosas de vértebras lumbares y de la musculatura paravertebral, Lasegue positivo a $20^{\circ}$ bilateral, paraparesia de extremidades inferiores y exaltación de los reflejos osteomusculares.
En la analítica realizada: $\mathrm{Hb}$ 9,6 g/dl, Hto 28,1\%, VCM 86,8 fl, VSG 47 mm/1 ${ }^{a}$ hora, GGT 189 UI/l, Fosfatasa alcalina 170 UI/l, LDH 665 UI/L, Beta2microglobulina 4,11 ng/ml, ECA normal.

Marcadores tumorales: Enolasa 27,42 mg/l, Alfafetoproteína normal. Mantoux negativo. Serologías (VEB, CMV, VHB, VHC, VIH y leishmania): negativas. Radiografía de tórax normal. En el TC toracoabdominal: adenomegalias axilares bilaterales. Adenomegalias perivasculares retroperitoneales pélvicas e inguinales. Líquido en espacio retroperitoneal. Hepatoesplenomegalia. En la RMN de columna vertebral se informó como proceso linfoproliferativo con afectación vertebral e intradural. Metástasis vertebrales múltiples (D10-D11-L1-L2-L3) con invasión de canal. Mielopatía compresiva a nivel de D11-D12 (Fig. 1). En gammagrafía con Galio 67, captación en adenopatías axilares y mediastínicas, hepatomegalia. En la biopsia de las adenopatías axilares el estudio anatomopatológico indicó linfoma de Hodgkin tipo celularidad mixta.

La paciente fue diagnosticada de linfoma de Hodgkin estadio IV tipo celularidad mixta y recibió tratamiento con quimioterapia con esquema ABVD desapareciendo de forma progresiva sus síntomas con disminución de las adenopatías y de la clínica de compresión medular recuperando la capacidad de deambulación.

La compresión medular puede ser la manifestación inicial de la enfermedad pero habitualmente es una complicación de las formas avanzadas del proceso $(4,5)$, sin embargo los linfomas con afectación medular son considerados como "primarios" $(1,6)$. La extensión ósea también es una situación poco frecuente en la enfermedad de Hodgkin (4), más todavía como localización primaria. Suele aparecer en personas de mediana edad como nuestra paciente aunque suelen ser varones1. El síndrome constitucional con fiebre alta e importante sintomatología también suele aparecer en fases tardías, y sobre todo en los tipos histológicos de celularidad mixta o depleción linfocítica.

En el caso de esta paciente dados sus antecedentes y origen se hizo diagnóstico diferencial con otros procesos que cursan con adenopatías y afectación medular/vertebral (tuberculosis, sarcoidosis, otros tumores, etc.) aunque el hecho de disponer de adenopatías accesibles para la biopsia y las características clínicas orientaron hacia el diagnóstico final.

El pronóstico del Hodgkin con esta localización suele ser malo dado el retraso diagnóstico al detectarse cuando las manifestaciones son ya evidentes $(2,5)$.

En cuanto al tratamiento la respuesta a la quimioterapia asociada o no a radioterapia es muy satisfactoria mejorando la clíni- 


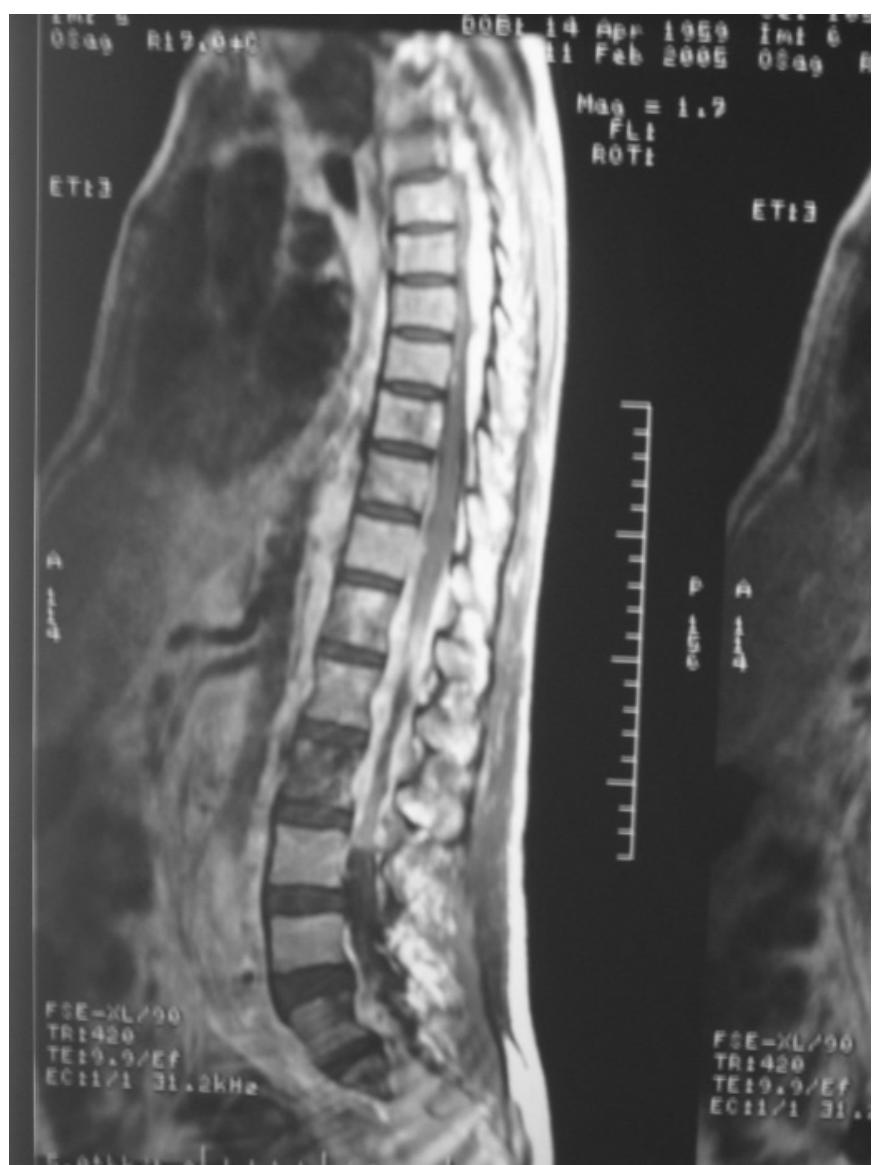

Fig. 1. Metástasis vertebrales múltiples de linfoma de Hodgkin con estenosis de canal.

ca precozmente, en el caso de no hacerlo se debe pensar en la descompresión quirúrgica urgente $(2,3)$.

J. Navarro Calzada, J. Cuesta Muñoz, M. J. Crusells Canales, S. Letona Carbajo, I. Sanjoaquin Conde, J. A. Amiguet García

Servicio de Enfermedades Infecciosas. Hospital Clínico Universitario "Lozano Blesa". Zaragoza

1. Chahal S, Lagera JER, Ryder J, Kleinschmidt-DeMasters BK. Hematologic neoplasms with first presentation as spinal cord compression síndromes: a 10-year retrospective series and review of the literatura. Clin Neuropathol 2003; 22: 282-90.

2. Toussaint D, Massagen N, Mathy L, Finet C, Vanderick J, Monseu G. Primary malignant lymphoma of the spine: clinical aspects and therapeutic advances. Acta Orthop Belg 1999; 65: 23-32.

3. Riffaud L, Adn M, Brassier G, Morandi X. Acute cauda equina compression revealing Hodgkin's disease. Spine 2003; 14: E270-E272.

4. Moridaira K, Handa H, Murakami H, Uchiyama T, Takeuchi T, Sato S, Tamura J, Naruse T, Tsuchiya J. Primary Hodgkin's disease of the bone presenting with an extradural tumor. Acta Haematol 1994; 92:148-149.

5. Lehman E, Anupindi S, Harris NL. A 15-year-old girl with pain in the left leg and back, pruritus and thoracic lymphadenopathy. N Eng J Med 2003; 348: 2443-51.

6. Di Marco A, Campostrini F, Garusi GF. Non-Hodgkin lymphomas presenting with spinal epidural involvement. Act Oncol 1989; 28: 485-8.
¿Son derivados en estadios precoces o avanzados los pacientes con cáncer a la consulta de oncología médica?

\section{Sr. Director:}

Una vez establecido el diagnóstico citológico o histológico de una enfermedad maligna, es preciso determinar la extensión, tanto local como a distancia, para de este modo evaluar las posibilidades terapéuticas y además poder realizar una apreciación del pronóstico del paciente. De este modo se puede asignar a cada paciente un código mediante el cuál se agrupa a los pacientes cuyos tumores tienen una extensión equivalente, un tratamiento similar y un pronóstico comparable.

Las clasificaciones deben ser sencillas y relativamente fáciles de comprender y de manejar. En la actualidad los enfermos que padecen enfermedades malignas se agrupan en un pequeño número de categorías, habitualmente cuatro, que se denominan estadios (1-3). A estadios más bajos el pronóstico es más favorable.

Esta forma de clasificación de los tumores es imprescindible puesto que reúne en cada estadio un grupo homogéneo de enfermos semejantes en principio, lo que nos permitirá indicar una aproximación al pronóstico de modo aproximado, facilitar la evaluación estadística de los resultados de los ensayos clínicos, intercambiar opiniones entre los diferentes grupos de trabajo y de este modo contribuir al avance de la investigación clínica (3).

Decidimos efectuar un estudio en el que incluimos los pacientes remitidos por primera vez a la consulta de Oncología Médica de nuestro hospital durante un período de tres meses. Analizábamos el tipo de tumor y el estadio en el que se encontraba en el momento de la derivación, así como datos de edad y sexo.

El número de pacientes incluidos fue 60 , por tipos de cáncer el más frecuente fue el cáncer de mama con 21 casos, seguido del carcinoma de colon y recto con 18, pulmón con 8 casos, ovario con 6 y estómago con 3 enfermos y otros cuatro casos de otros tumores.

El cáncer de pulmón fue excluido del estudio al observar que los pacientes con cáncer de pulmón no microcítico que nos eran derivados, estaban incluidos en los estadios III y IV, por lo que los estadios más bajos, que fueron tratados con cirugía, no llegaban a la consulta de oncología médica de nuestro centro.

También excluimos del estudio los cuatro enfermos con tumores muy poco frecuentes, por lo que únicamente incluimos en el estudio a 48 pacientes.

De las 21 pacientes con cáncer de mama, 15 estaban incluidas en los estadios I y II, 4 en el III y 2 en el IV. Por lo tanto tenían enfermedad metastásica en el momento de la derivación un 9,5\% de las enfermas y enfermedad localmente avanzada un $19 \%$.

En conjunto el cáncer de mama fue derivado en estadio III o IV en un $28,5 \%$ de los casos, lo que nos parece bastante elevado.

Con respecto al cáncer de colón o recto, fueron derivados en estadio I ó II, 9 de 18 enfermos (50\%), en estadio III, 6 pacientes $(33,3 \%)$ y en estadio IV, 3 enfermos (16,6\%). Por lo tanto tenían enfermedad localmente avanzada o metastásica en el momento de la derivación un 50\% de los casos, lo que también consideramos muy elevado.

Los pacientes derivados diagnosticados de cáncer gástrico, estaban todos en estadio IV (100\% de los casos), lógico en parte, porque sin duda, un número más elevado de casos en estadios más precoces no habrán sido derivados desde cirugía a nuestra consulta, al no tener un papel excesivamente relevante la quimioterapia en los estadios más precoces.

Con respecto al cáncer de ovario, de las 6 pacientes derivadas, había 4 en estadio I, una en estadio III y otra en estadio IV. Por lo tanto un 33,3\% estaban en estadios localmente avanzados o metastásicos. Sin embargo hay que recordar que en el cáncer de 